\title{
Opinion \\ The Present and the Future Works on the Algae's Valorization for Production of our Future Energies?
}

Volume 5 Issue 5 - 2017

\section{Opinion}

This question is recurrent but important to implement the work of program forms and the efforts of the different implicated actors to guarantee a future for the next generations. Some questions and some reflections are made and detailed in these pages. Many studies based on the raw data obtained during field investigations have evaluated the human population and occidental food interests among others. They have shown an occidental population awareness for the nutritive quality of the algae. The interest growth arouses the personal mobilisation on the field trip to realize the picking of the marine plants. The identification of these organisms is not trivial and needs some efforts arouses by an interest for the well being. Moreover, the algae utilization in different domains are not longer to be proved.

First, algae, is a photosynthetic and autotrophic organism, which draws its energy from the natural or artificial lights. Since this organism has been autrotrophic and then is able to assimilate the atmospheric carbon dioxyde (CO2 chemical form): this "passed plant" is an amazing assimilative plant of atmospheric carbon and water solved carbon. This mechanism is dependent completely on a enzyme presents in different molecular forms and presents in different kind of algae tissue and cellular organ its. It deals with a protein named carbonic anhydrase which is capable to transform the carbon dioxyde into bicarbonate with reversible biochemistry reaction. These algae present at the various depth in the cost and littoral areas could be, as a moderator and then lessen the acidification process known (because measured) of the marine water. This acidification phenomenon comes from the atmospheric $\mathrm{CO} 2$ increasing generated by the fossil energy combustions.

Then we have to present the energy precious and essential to the actual humanity which is generated from the physic, the chemistry and the biochemistry... During the decades passed we used the fossil forms of algae called oil to advance the technologic progress and the economy world. We can maybe already saying that the accelerating process in laboratory is relevant, because these events to produce fossil energy needed many billions years. These new energy made in lab and handmade creating new jobs, is used in the technologies known as the engines of the planes, of the cars.

Currently, various projects were and for other are trying to set up and for some of them have allowed to lead to the development of the fuel coming from the algae and particular from micro-algae. These micro-organisms have been isolated from various geographic areas, keeped and studied in laboratories. Most of them have assets to become a interesting strains in the recoverable and high-light biomolecules: interesting, in other terms, qualitatively and quantitatively exploitable.

The algae is as well, an unicellular organism for the microscopic form or a few organs for the macroscopic one, and considered as a bio-molecule firm. These biological "firms" made the bio-molecules with chemical elements coming from the Earth original formation

\author{
Moulin Pascale \\ Aix-Marseille II University, France
}

Correspondence: Moulin Pascale, Aix-Marseille II University, France, Tel 0033-677676986,Email Pascale.moulin@laposte.net

Received: May 07, 2017| Published: May 09, 2017

( $-4,5$ billion years): the carbon, the nitrogen, some particules (as iron, aluminium, silicone...) and the water with the energy light. In the particular case, the blue algae (cyanobacteria) ables to fix the diazote making it the pioneer of life ( -4 billion years). In the other case, the diatoms participate to the silica ocean cycle, chemical element coming from the magma energy earth. This apparent simplicity in the chemical needs produce, without human influences, molecules set from original metabolisms because not very well-known.

Why do we have some interests to produce these molecules made from this biomasses? Classically, they are valorized in cosmetic, biodegradable materials, agriculture. For a while, new applications could be appear in the occidental foods "animal" because the "animals" microbiote is studies subject for the health "animal" stade. This bacteria flora is variable from one individual animal to another one. The algae seems interesting certainly in nutrititional point of view and intervenes in the microbiote quality.

We have highlighted in some cases bio-molecules (proteins, pigments, lipids) can be produced in higher or small amounts depending of the induction or repression of their metabolisms. These metabolisms have played in the adaptation of the organisms face to the environmental changes (salinity, nutriments, temperature, light) which could be considering in the Future. These molecules valorized are different from the higher plants and they can be produce in culture conditions controlled. For example, "blue" algae (Spirulina) farms are setting up in the continent in the controlled ways.

In conclusion, are these resources inexhaustible? The balance sheet is the following that some geographical area are rich naturally in algae biomasses and biodiversity and other one are cultivated surface with algae directly economically recoverable. Some algae are conserved in laboratory, manipulated below various culture conditions. Some of them are "stressed" during experimental process. And some results have shown in that controlled environment these organisms were putting in place some changement of molecular replies to be adapted to the new conditions. These ancient/past plants have been gone through the ages under the environmental changes like new climatic, atmospheric, light, nutriment, temperature parameters they have been 
showing a phenotype and genotype plasticity for these changes. These different marine organisms could have still a long future.

\section{Acknowledgments}

None.

\section{Conflicts of interest}

None. 